\title{
A robot-based behavioural task to quantify impairments in rapid motor decisions and actions after stroke
}

Teige C. Bourke ${ }^{1 *}$, Catherine R. Lowrey ${ }^{1}$, Sean P. Dukelow ${ }^{5}$, Stephen D. Bagg ${ }^{2}$, Kathleen E. Norman ${ }^{1,3}$ and Stephen H. Scott ${ }^{1,4}$

\begin{abstract}
Background: Stroke can affect our ability to perform daily activities, although it can be difficult to identify the underlying functional impairment(s). Recent theories highlight the importance of sensory feedback in selecting future motor actions. This selection process can involve multiple processes to achieve a behavioural goal, including selective attention, feature/object recognition, and movement inhibition. These functions are often impaired after stroke, but existing clinical measures tend to explore these processes in isolation and without time constraints. We sought to characterize patterns of post-stroke impairments in a dynamic situation where individuals must identify and select spatial targets rapidly in a motor task engaging both arms. Impairments in generating rapid motor decisions and actions could guide functional rehabilitation targets, and identify potential of individuals to perform daily activities such as driving.
\end{abstract}

Methods: Subjects were assessed in a robotic exoskeleton. Subjects used virtual paddles attached to their hands to hit away 200 virtual target objects falling towards them while avoiding 100 virtual distractors. The inclusion of distractor objects required subjects to rapidly assess objects located across the workspace and make motor decisions about which objects to hit.

Results: As many as $78 \%$ of the 157 subjects with subacute stroke had impairments in individual global, spatial, temporal, or hand-specific task parameters relative to the $95 \%$ performance bounds for 309 non-disabled control subjects. Subjects with stroke and neglect (Behavioural Inattention Test score <130; $n=28$ ) were more often impaired in task parameters than other subjects with stroke. Approximately half of subjects with stroke hit proportionally more distractor objects than $95 \%$ of controls, suggesting they had difficulty in attending to and selecting appropriate objects. This impairment was observed for affected and unaffected limbs including some whose motor performance was comparable to controls. The proportion of distractors hit also significantly correlated with the Montreal Cognitive Assessment scores for subjects with stroke $\left(r_{s}<=-0.48, P<10^{-9}\right)$.

Conclusions: A simple robot-based task identified that many subjects with stroke have impairments in the rapid selection and generation of motor responses to task specific spatial goals in the workspace.

Keywords: Stroke, Assessment, Cognitive impairments, Attention, Inhibition, Neglect

\footnotetext{
* Correspondence: 8tb36@queensu.ca

${ }^{1}$ Centre for Neuroscience Studies, Queen's University, Kingston, ON, Canada

Full list of author information is available at the end of the article
} 


\section{Background}

Moving and interacting in the world requires rapid processing of the visual environment to identify potential motor goals, select a movement and finally move in a timely manner. For example, when packing groceries, we must decide where to put items based on their shape, size, fragility and other features. The selection, planning and execution of motor actions must be done rapidly to keep pace with the flow of groceries from the cashier.

There is growing evidence that sensory feedback is rapidly integrated into motor decisions [1-3]. Sensory feedback is integrated with higher-level behavioural goals to make rapid decisions on how to move and interact in the environment. Selective attention refines spatial representations of the environment into potential movement targets $[1,4]$. The choice between these internal representations is then based on 'decisional factors' [1]. One such factor is the recognition of combinations of visual features and their behavioural relevance $[1,5,6]$. Thus, the sensorimotor system rapidly integrates information on the environment to guide motor decisions.

Another important aspect of voluntary motor control is the ability to inhibit a motor action [7]. When instructed, it is very automatic to simply reach towards spatial targets as they appear in the workspace [8]. In contrast, it can be hard to avoid reaching towards a target when instructed to move in the opposite direction. In this anti-reach condition, subjects can make erroneous initial motor responses to the spatial goal and are delayed in moving in the opposite direction. This task requires the voluntary override of an automatic response to reach towards the target and involves many brain areas including frontal and parietal cortex [7, 9-12]. This ability can be impaired in persons with stroke [12], mild cognitive impairment [13], Alzheimer's Disease [14], and a history of concussion [15], Thus, successful voluntary motor control involves processing sensory feedback not only to select motor actions but also to avoid making others.

Post-stroke disability stems from a variety of motor, sensory, and/or cognitive deficits [16]. The ability to pack groceries described above highlights that impairments in these functional tasks may reflect not only motor impairments but also cognitive impairments. When driving, one must quickly decide on actions to apply pressure to the brake or accelerator pedals, or turn the wheel based on information from street signs, traffic signals, other traffic, and pedestrians. However, neuropsychological tests or cognitive screening tools generally separate motor and cognitive assessments - the latter often requiring verbal or written responses - and typically do not impose time limits to perform the tasks $[17,18]$. Few neuropsychological assessments focus on rapid motor decisions beyond simple reaction time tests $[18,19]$, or timed cognitive tasks such as trail making [20], even though complex and time sensitive demands are often required for everyday activities.

Furthermore, many standard assessments of post-stroke functioning have problems of subjectivity, coarse ordinal scales, criteria-based scoring, and lack of responsiveness (including floor and ceiling effects) [21]. Thus, we developed a novel approach of using a robotic assessment to provide objective, continuous measures of performance that are compared to a normative model of healthy control performance.

We recently used an object hit task to quantify simultaneous upper limb bimanual sensorimotor performance [22]. Although this task quantified rapid motor skills, decisional processes required to perform the task were limited to identifying the trajectory of an object and selecting a limb to hit the object. As all objects were targets in this task, it did not require cognitive processes related to attending to object qualities (rather than just spatial location) to select a motor action nor require inhibiting inappropriate motor responses. These processes can be impaired following stroke $[9,23,24]$.

The goal of the present study was to develop a task that examined rapid motor skills with both arms that also required greater cognitive processing. We developed a variant of the object hit task [22] by requiring subjects to hit 2 possible targets while avoiding all other objects in the workspace. We hypothesize that individual subjects with stroke will be impaired in enacting or inhibiting a motor response to a potential target based on sensory feedback of the object's features and their relevance to the ongoing task. The performance of subjects with stroke was compared to a large cohort of non-disabled control subjects.

Clinically, knowledge of impairments in these more complex visuomotor skills can guide novel rehabilitation strategies to regain the ability to rapidly process sensory information for motor actions. As well, it may help to identify if individuals should return to more complex daily activities such as driving.

\section{Methods \\ Subject information}

Participants included patients recruited from Providence Care (St. Mary's of the Lake Hospital, Kingston, ON), the Dr. Vernon Fanning Centre and Foothills Hospital (Calgary, AB). Prospective subjects were excluded if they had other significant neurologic diagnoses (e.g., Parkinson's disease), acute medical illness, and/or ongoing upper extremity musculoskeletal injuries. Subjects were also excluded if they appeared fatigued, reported pain associated with attempting robotic assessments or reported pain during clinical testing on strength or range of motion that would be relevant to the robotic task. Non-disabled control subjects were recruited from the Kingston, ON and Calgary, $\mathrm{AB}$ communities. This study was approved 
by the Queen's University Health Sciences and Affiliated Teaching Hospitals Research Ethics Board (\#ANAT-02405) and the University of Calgary's Conjoint Health Research Ethics Board (\#22123) and subjects provided informed consent.

\section{Experimental setup}

Details of the robotic set-up have been reported previously $[25,26]$. Briefly, the behavioural task was performed using a bimanual exoskeleton robot which measures limb motion (KINARM, BKIN Technologies Ltd, Kingston, ON, Canada). Participants sat in a modified wheelchair base, and their arms were fitted in supports permitting movement in the horizontal plane. Arm supports were adjusted such that the robot's linkages aligned with the subject's elbows and shoulders. Subjects received visual feedback from a virtual reality system which displayed fingertip position and virtual objects in the same plane as arm motion via a two-way mirror. Direct vision of the hands and arms was occluded.

\section{Behavioural task}

Subjects were assessed in an object hit and avoid task (Fig. 1a), which is based on a previous object hit task [22]. At the beginning of the task, subjects were presented two shapes on the screen. Subjects were instructed to hit these two shapes ('targets') away from them and avoid all other shapes ('distractors'). Subjects could use both hands which were represented by horizontal paddles. Both target objects and distractor objects dropped from one of 10 locations along the top of the screen $8 \mathrm{~cm}$ apart (virtual bins). A total of 30 objects (20 targets and 10 distractors) were released at each bin (200 targets and 100 distractors total). Objects were released from all 10 bins before a bin was reused. Objects dropped at an increasing rate following the equation:

$$
\begin{aligned}
\text { Drop Rate }= & 0.5 \text { objects } / \text { second } \\
& +\left[0.025 \text { objects } / \text { second }^{2} \times(\operatorname{time}(\mathrm{s}))\right]
\end{aligned}
$$

The maximum number of objects possible to appear on the screen simultaneously increased from 1 to 16 over the course of the task. The speed of the objects moving towards the subject was 50 to $100 \%$ of maximum drop speed, which increased following the equation:

$$
\max \text { drop speed }=15 \mathrm{~cm} / \mathrm{s}+\left[0.3 \mathrm{~cm} / \mathrm{s}^{2} \times(\operatorname{time}(\mathrm{s}))\right]
$$

Thus targets moved at $\sim 10 \mathrm{~cm} / \mathrm{s}$ in the beginning of the task and increased to $\sim 50 \mathrm{~cm} / \mathrm{s}$ by the end of the task. Position of the objects and hand position were recorded at $200 \mathrm{~Hz}$. The task took just over $2 \mathrm{~min}$ to complete.

One of 6 task variants were used with varying shapes designated as targets and distractors. Target pairs had similar width but were always different heights and

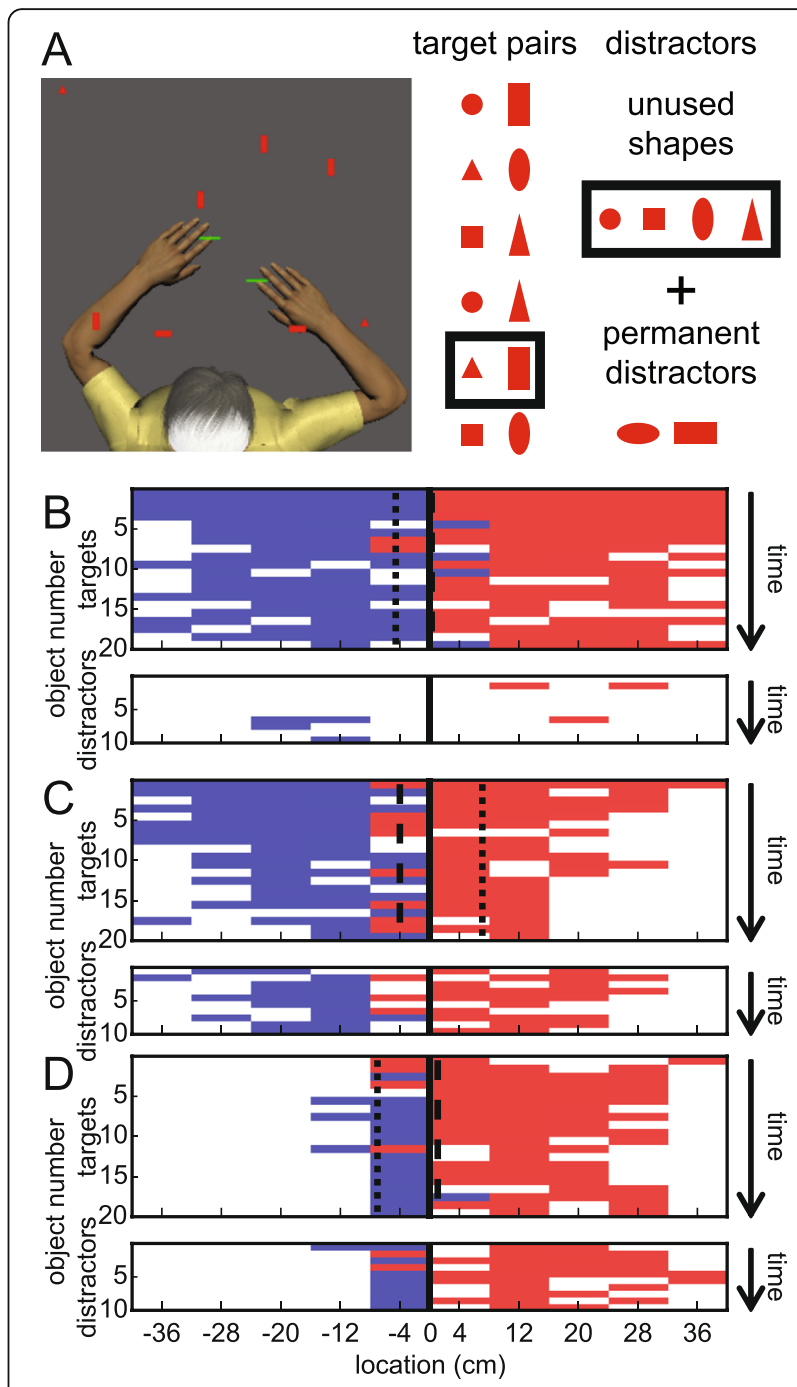

Fig. 1 Task details and exemplar subjects. a Screenshot of a subject performing the task. Objects included 2 target shapes (chosen from 6 pair variants) and 6 distractor shapes ( 4 are shapes used as targets in other task variants and 2 were always distractors). b Task performance summary of a 62 year old right-handed male control subject. $Y$ axes are number of targets (top) or distractors (bottom) dropped from each bin ( $X$ axis). Hits with the left hand are blue areas and hits with the right hand are red areas. Missed objects are the white areas. The top of each plot represents the beginning of the task, and the bottom represents the end. Hand transition and miss bias are indicated with dashed and dotted lines (respectively). c Performance of a 65 year old right-handed, right-affected male subject 5 days post-stroke. d Performance of a 63 year old right-handed male subject 8 days post-stroke. Subject was left-affected and had a BIT score of 67 (indicative of visual neglect)

different classes of shapes (Fig. 1a). Distractors consisted of the remaining unused shapes (shapes used as targets in other task variants), as well as two wider shapes.

Every effort was made to ensure subjects understood the task instructions. Operators usually obtained verbal confirmation that they understood which targets to hit 
when showing the target objects before starting the task. Reminders to hit the specific target shapes and avoid all others would be given early in the task, especially if there seemed to be confusion with similar distractor shapes (for example tall target rectangle vs. wide rectangle distractor). As well, targets hit by a paddle were knocked away and haptic feedback of the contact was provided by the robot [22], whereas distractors simply passed through the paddle to provide immediate feedback that it was a distractor.

\section{Data processing}

Data were analyzed using MATLAB (Mathworks Inc., Massachusetts). Hand speed was filtered using a sixth-order double-pass Butterworth filter (cutoff frequency $10 \mathrm{~Hz}$ ).

\section{Tasks parameters}

We used 14 metrics to quantify task performance in order to characterize a diverse range of sensory, motor and cognitive functions examined in this task. Most of the parameters paralleled the metrics in our previously published object hit task [22], a simpler version of this task in which there were no distractors. As well, a few parameters were added or modified to capture the addition of distractors in the present task.

Global Performance was evaluated using five parameters:

1. Targets hit: The number of target objects hit away from the body.

2. Distractors hit: The number of distractor objects hit.

3. Objects hit: The number of objects hit (target hits + distractor hits).

4. Distractor proportion: Distractors hit divided by objects hit.

In the case of multiple hits for the same object, the first hit is used to determine which hand/paddle hit the ball.

5. Object processing rate (objects/second): The rate of correctly processed objects (number of targets hit + distractors missed per second) at $80 \%$ of task completion. The rate of correctly processed objects was determined at every time step (every $0.005 \mathrm{~s}$ ) from the time the first object was hit or left the screen to the time the $240^{\text {th }}$ object ( $80 \%$ of task complete) was hit or left the screen. To filter this signal, we convolved the rate with a Gaussian window (MATLAB function normpdf). From this rate signal, the optimal growth curve $(\mathrm{y}=<\max$ height $>*(1-\exp (-(<$ curvature $>) *<$ data $>)))[27,28]$ for the data was calculated and the value of this curve when $80 \%$ of the task was completed was used to approximate the maximal object processing rate for each individual subject. The rate was taken at $80 \%$ of task complete so that performance was at or near maximum, but not at $100 \%$ as the ratio of distractor object dropping statistically increased at the end of task. This is because there is always a $66 \%$ chance of dropping a target object, but objects are sampled without replacement, leading to the statistical scenario of running out of target objects and only being able to drop distractor objects from a given bin at the end of the task.

All other parameters were defined in the same way as the object hit task [22].

Spatial and Temporal Performance

6. Miss bias: Spatial position quantifying the extent to which the number of target misses deviates from being equally distributed on either side. Computed as sum of target misses in each bin (m), multiplied by the bin position ( $\mathrm{x}$ ), and then divided by the total number of target misses $(\operatorname{sum}(\mathrm{mx}) / \operatorname{sum}(\mathrm{m}))$. Given that the centre of the bins is $x=0$, the greater the mean location of misses deviates from 0 , the more misses were on the left or right side of the workspace (dependent on whether the miss bias is negative or positive, respectively).

7. Hand transition: Spatial transition point in subject's hand preference for hitting targets. This is the mean of the right hand's and the left hand's weighted means of their respective target hit distributions. The weighted mean of each hand only includes target hits by that hand in bins where both hands have been used to hit targets (overlapping bins) and one additional bin beside the overlapping bins (where that hand has been used to hit targets). If bins do not overlap, only the leftmost bin with target hits from the right hand and the rightmost bin with target hits from the left hand are used in the weighted means.

8. Median error (\% of targets): Point in time when subjects missed half of the target objects that they missed over the entire task.

\section{Hand Specific Performance}

9. Movement Area: The areas of space used by each hand during the task. Computed as the area of the convex hull- a complex polygon which captures the boundaries of the movement trajectories of each hand [22, 29]. Calculated for each hand separately.

10.Hand speed: The average hand speed calculated from each time step ( $5 \mathrm{~ms}$ ) over the course of the task. Calculated for each hand separately.

11.Hand bias hits: The difference between the number of target hits with the right hand and the number of 
target hits with the left hand divided by the total number of target hits.

12.Hand selection overlap: The number of times successive target hits from a given bin were with different hands divided by the total number of target hits.

13.Hand movement bias area: Difference in movement area of the right and left hands divided by the sum of the movement area of the right and left hands.

14.Hand bias speed: The difference in mean hand speed of the right and left hands divided by the sum of the mean hand speed of the right and left hands.

\section{Statistical analysis}

Performance of control subjects was analyzed for any effects of age, sex, or handedness. Control values were ageregressed and Box Cox transforms were used to normalize control distributions when necessary [30, 31]. Control parameter values were then assessed for any effect of sex or handedness, and values subdivided into respective categories if effects were significant, and age regressed and Box Cox transformed again if necessary. All parameter values were converted to z-scores of the model to allow for comparison across all subjects (because age, sex, and handedness are now accounted for in the model). Individual subjects with stroke were defined as having impaired performance on a task parameter, when their z-score was $>1.65$ or $<-1.65$ for one tailed tests, or $>|1.96|$ for two tailed tests.

A subset of subjects was assessed a second time by a different operator within 7 days of their initial assessment. An intraclass correlation was used to determine interrater reliability (significant if $P<0.05$, acceptable if ICC $>0.8$ ).

\section{Clinical assessments}

Subjects with stroke were evaluated by a trained physician, physiotherapist, or occupational therapist using a number of standardized clinical assessments. Both arms were assessed using the Chedoke-McMaster Stroke Assessment (CMSA) [32] to determine arm function. The CMSA is based on Brunnstrom's stages of motor recovery poststroke [33]. Subjects were broadly categorized as "Left-Affected" (LA) or "Right-Affected" (RA) depending on the clinically most affected side of the body. Elbow flexor spasticity was measured by the Modified Ashworth Scale, which categorizes the amount of resistance produced by the arm in response to passively moving it through its range of motion [34]. Functional abilities were measured with the Functional Independence Measure (FIM), which has both a motor and cognitive component [35]. The conventional subtests of the Behavioural Inattention Test (BIT) were used to screen for deficits in spatial attention [36]. Subjects with stroke who scored $<130$ on the BIT were defined as having visuospatial neglect and were analyzed separately (neglect subjects). Subjects were also screened for cognitive deficits using the Montreal Cognitive Assessment (MoCA)
[37]. The handedness of controls and subjects with stroke was determined by the Modified Edinburgh Handedness test [38].

\section{Results \\ Subject demographics and clinical information}

Table 1 shows the demographic information and clinical scores for the 157 subjects with stroke and 309 control subjects. The majority of subjects with stroke were assessed early, with only 17/157 subjects being assessed $>28$ days of their stroke. Exclusion of these 17 subjects did not substantively alter the present results. Subjects with stroke were usually assessed either on the same day $(n=90)$ or within 1 day $(n=40)$ of the robotic assessment. Some subjects with stroke were assessed within $2-4$ days $(n=18)$ and a few within 5-10 days $(n=9)$. Twenty eight subjects with stroke displayed visual neglect as indicated by scores of $<130$ on the BIT. These subjects were analyzed separately to assess differences in the patterns of task performance with stroke and visual neglect.

\section{Exemplar subjects}

Figure 1b-d displays the distribution of target and distractor hits and misses for a control subject and two subjects with stroke. The control subject (Fig. 1b) was very effective in hitting targets (136/200) and avoiding distractors (94/100). Control subjects gradually missed more targets, especially lateral ones, as task difficulty increased. The RA subject with stroke (Fig. 1c) hit fewer targets $(117 / 200)$ and more distractors $(47 / 100)$ than the control. The LA neglect subject (Fig. 1d) hit even fewer targets (83/200) and a similar amount of distractors (40/ 100). This subject also hit very few objects with their left hand and very few on the left side of the workspace.

\section{Impairments identified using the robot-based task}

Each parameter classified a varying number of subjects with stroke as impaired (Table 2). Target hits identified the largest number of subjects as impaired. The number of targets hit by controls depended on age and sex (Fig. 2a). Subjects with stroke were considered to be impaired in targets hit if their performance fell below the $5 \%$ level performance of controls after correcting for age and sex. Overall, $78 \%$ of LA subjects (left-affected subjects with stroke), $68 \%$ of RA subjects (right affected subjects with stroke), and $96 \%$ of neglect subjects (subjects with stroke and visual neglect) were impaired in targets hit. Similarly, age effects were found for objects hit. In total, $64 \%$ LA subjects, $51 \%$ RA subjects, and $86 \%$ of neglect subjects hit less objects than the lower cutoff of the age normative model.

Distractor proportion identified more individual subjects with stroke as impaired (39 \% LA, 51 \% RA, 79 \% neglect; see Fig. $2 \mathrm{~b}$ and Table 2) compared to distractors hit (15\% 
Table 1 Demographic information of subjects included in the experiment

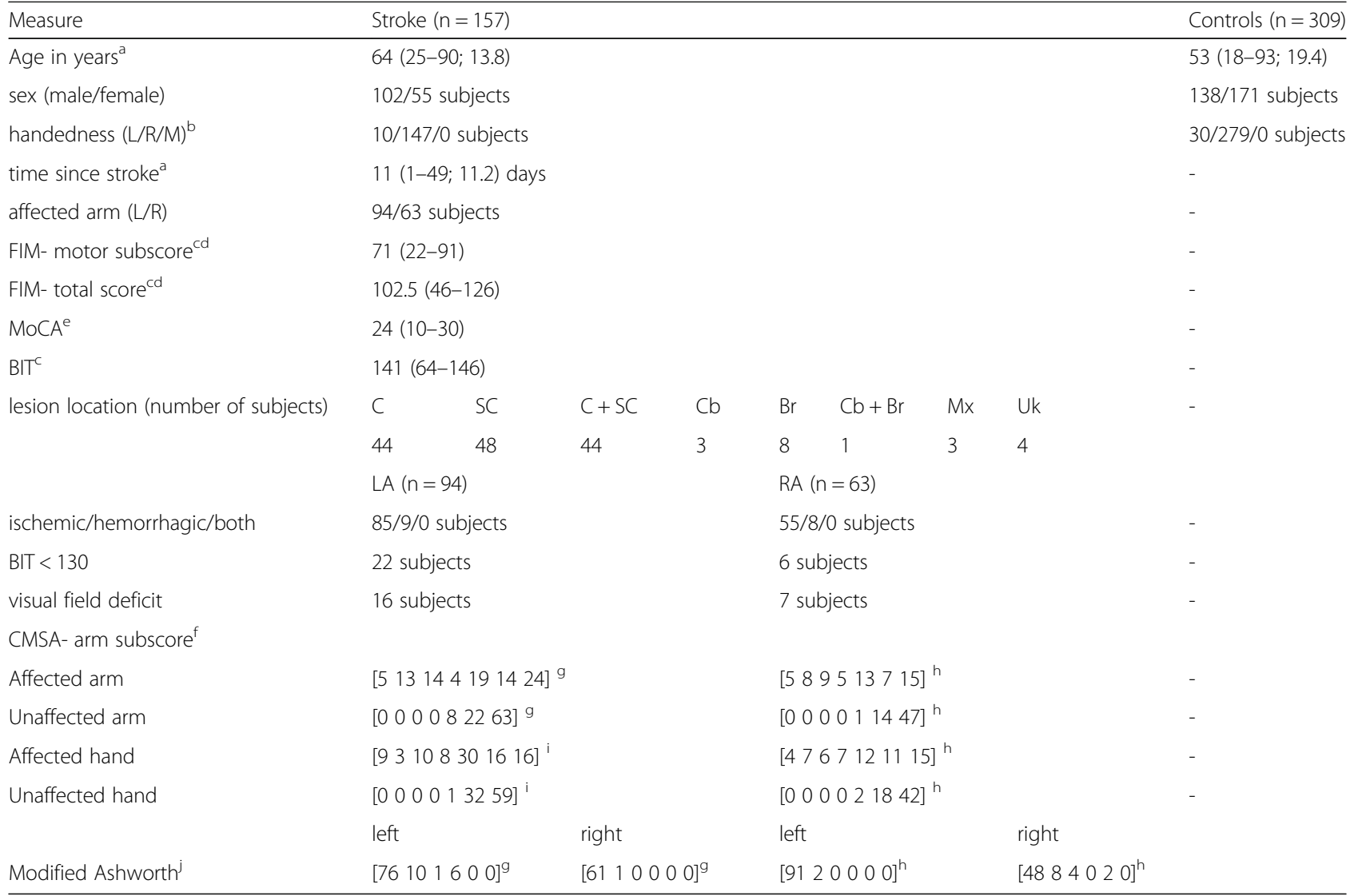

Abbreviations: $L / R / M$ (left/right/mixed), FIM (functional independence measure), MoCA (Montreal cognitive assessment), $B I T$ (behavioural inattention test), LA (left affected), $R A$ (right affected), $C$ (cortical), SC (subcortical), C +SC (cortical + subcortical), $C b$ (cerebellar), $B r$ (brainstem), $C b+B r$ (cerebellar + brainstem), $M x$ (mixed), Uk (unknown), CMSA (Chedoke-McMaster Stroke Assessment)

Legend: ${ }^{\mathrm{a}}$ median (min-max; standard deviation). ${ }^{\mathrm{b}}$ Handedness as determined by the Edinburgh Handedness Test. ${ }^{\mathrm{c}}$ median $(\min -\mathrm{max}) .^{\mathrm{d}} \mathrm{n}=156 .{ }^{\mathrm{e}} \mathrm{n}=152 .{ }^{\mathrm{f}}$

[n1 n2 n3 n4 n5 n6 n7] corresponds to the number of subjects with CMSA subscores of [1 234456 7]. ${ }^{9} n=93 .^{h} n=62 .^{i} n=92 .^{j}$ [n1 $n 2 n 3 n 4 n 5 n 6$ ] corresponds to the number of subjects with Modified Ashworth scores of [0 $111+234]$ for elbow flexion

of subjects with stroke impaired). Object processing rate was also a parameter that identified a large proportion of subjects with stroke (Fig. 2c): controls mostly had a processing rate between 1.5 to 3 objects per second, whereas the object processing rate of most subjects with stroke was below 2 .

Subjects with stroke who hit fewer objects also tended to hit a higher proportion of distractors (Spearman correlation; controls: $r_{s}=0.16, P=0.006$; subjects with stroke: $\left.r_{s}=-.33, P=3 \times 10^{-5}\right)$. Twenty nine percent of subjects with stroke displayed impairments in both object hits and distractor proportion (Fig. 2d, upper left quadrant). In contrast, $29 \%$ of subjects with stroke had impairments in only object hits (Fig. 2d, lower left quadrant) and $16 \%$ had impairments in only distractor proportion (Fig. 2d, upper right quadrant). All neglect subjects were impaired in at least one of these two parameters, and $64 \%$ were impaired in both parameters.

Almost all subjects with stroke (92\%) hit fewer objects with their affected arm than with their unaffected arm (Fig. 3a). Similarly, $77 \%$ of subjects with stroke showed a greater distractor proportion with their affected arm than with their unaffected arm (Fig. 3b). Overall, $57 \%$ of LA subjects, $60 \%$ of RA subjects, and $50 \%$ of neglect subjects had impaired object hits with their affected arm only.

Motor and distractor-related impairments in performance of the affected arm commonly co-occurred. Of the subjects whose affected arm was impaired in distractor proportion, the same arm was usually also impaired in objects hit (97 \% LA, $81 \%$ RA) and/or hand speed (85\% LA, $63 \%$ RA) (Fig. 3c). Distractor proportion was also negatively correlated with the number of object hits by the affected arm of subjects with stroke $\left(r_{s}=-0.58, P<10^{-10}\right)$. Although this would be expected, a negative correlation was not observed in the non-dominant arm of controls $\left(r_{s}=0.15, P=0.008\right)$.

In contrast, this coupling of motor and distractor-related impairments was less common for the unaffected arm. For the subjects with stroke who had impaired distractor proportion with their unaffected arm, the majority did not have impaired object hits (47\% LA, $82 \%$ RA) or hand speed (58 \% LA, $75 \%$ RA) with the same arm (Fig. 3d). The correlation between distractor proportion and objects 
Table 2 Task performance, interrater reliability, and clinical correlations. Task parameter sensitivity is defined by the corresponding z-score cutoff range

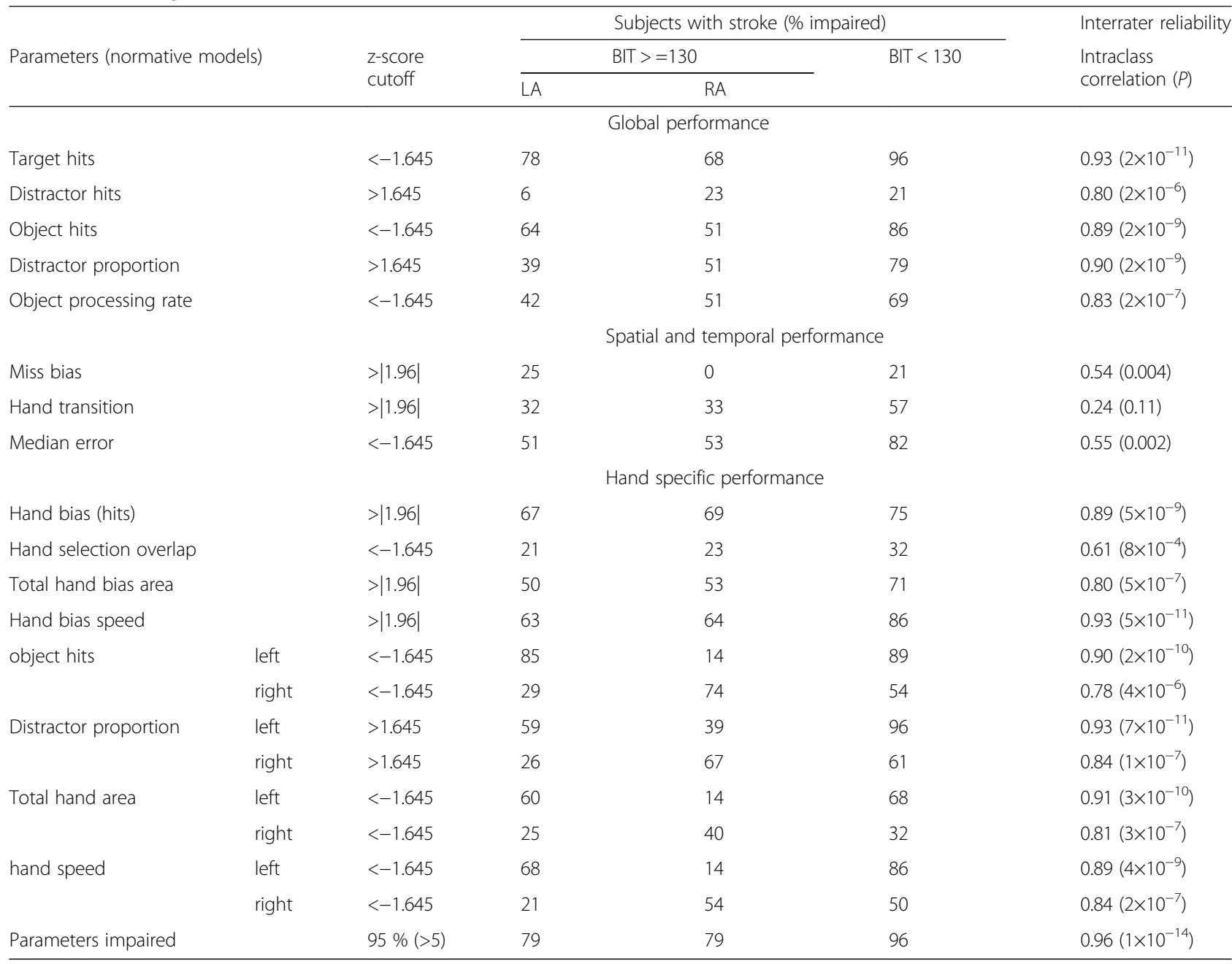

Abbreviations: BIT (behavioural inattention test), LA (left-affected subject), RA (right-affected subject)

Interrater reliability is shown by the intraclass correlation and corresponding $p$-values

hit was weaker for unaffected arm performance of subjects with stroke $\left(r_{s}=-0.15, P=0.07\right)$. A negative correlation was not observed for the dominant arm of controls $\left(r_{s}=0.18, P=0.001\right)$.

Neglect subjects who had impaired distractor proportion with their affected arm were usually impaired in hitting objects and/or hand speed with the same arm (100 and $92 \%$ impaired in both parameters, respectively). Impairments in distractor proportion for their unaffected arm were less likely to co-occur with impairments in objects hit and/or hand speed with that arm (36 and $36 \%$ impaired in both parameters, respectively).

We aggregated the number of task parameters that each subject was impaired in (Table 2). Most subjects with stroke $(82 \%)$ were impaired in more task parameters than $95 \%$ of controls ( $>5$ parameters). This included all but one subject with neglect.

\section{Correlations with standard clinical assessments}

Task parameter measures were compared to scores on the FIM, MoCA, and BIT (Table 3). Object hits showed moderate correlations with BIT $\left(r_{s}=0.40, P=2 \times 10^{-7}\right)$ and FIM scores $\left(r_{s}=0.45, P=3 \times 10^{-9}\right)$ and weak correlations with MoCA scores $\left(r_{s}=0.23, P=0.004\right)$. Distractor hits displayed modest correlations with MoCA $\left(r_{s}=-0.31, P=\right.$ $\left.1 \times 10^{-4}\right)$, but distractor proportion displayed moderate correlations with BIT $\left(r_{s}=-0.43, P=2 \times 10^{-8}\right)$, FIM $\left(r_{s}=-0.45\right.$, $\left.P=4 \times 10^{-9}\right)$, and MoCA $\left(r_{s}=-0.49, P=2 \times 10^{-10}\right)($ Fig. $4 \mathrm{a}, \mathrm{b})$. Out of the $36 \%$ of subjects with stroke who passed the MoCA (scored > =26), $27 \%$ of these subjects with stroke were impaired in distractor proportion. Object hits with the affected arm correlated better with the FIM $\left(r_{s}=0.51\right.$, $\left.P=1 \times 10^{-11}\right)$ than the unaffected arm $\left(r_{s}=0.26, P=8 \times 10^{-4}\right)$. Object hits with the unaffected arm showed a modest correlation with BIT $\left(r_{s}=0.30, P=1 \times 10^{-4}\right)$ and a weak correlation with MoCA $\left(r_{s}=0.17, P=0.04\right)$. Distractor 

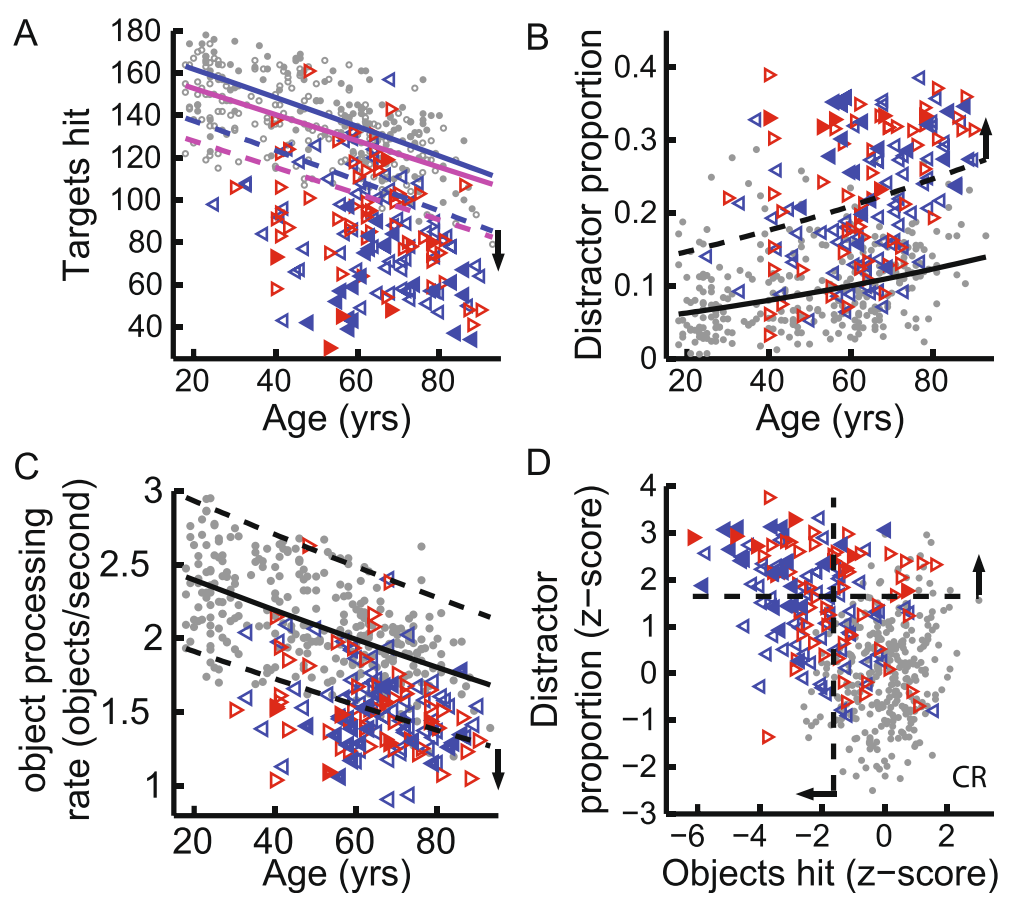

Fig. 2 Global Performance in Task Parameters. a Scatter plot of age versus target hits. Performance of male and female controls is shown by filled and empty grey markers, respectively. Performance of subjects with stroke is shown by the leftward and rightward pointing triangles representing left-affected and right-affected subjects, respectively. Triangle markers are filled if subject also had a BIT score $<130$ indicative of visual neglect. Age normative model is shown by the blue and magenta lines representing the median (solid lines) and cutoff (dashed lines) z-score for male and female control subject performance distribution (respectively) according to the model. The black arrow indicates which side of the cutoff score corresponds with subjects being impaired on the particular parameter. b Scatter plot of age versus distractor proportion. Performance of control subjects is shown by the filled grey markers. Age normative model is shown by the median and cutoff z-score of control subject performance distribution according to the model. c Scatter plot of age versus estimated maximum object processing rate. $\mathbf{d}$ Scatter plot of object hits versus distractor proportion. Values have been converted to z-scores based on the normative models. Dashed lines represent the cutoff used to indicate impairment in each parameter. The control performance range is the quadrant indicated by the 'CR'

proportion with the unaffected arm showed moderate correlations with $\operatorname{BIT}\left(r_{s}=-0.41, \quad P=9 \times 10^{-8}\right)$ and MoCA $\left(r_{s}=-0.48, P=4 \times 10^{-10}\right)$. MoCA scores correlated most strongly with overall and unaffected arm distractor proportion $\left(r_{s}=<=-0.48, P<10^{-9}\right)$. The number of parameters impaired was also moderately correlated with FIM $\left(r_{s}=-0.61, P=2 \times 10^{-17}\right)$ and BIT $\left(r_{s}=-0.43\right.$, $\left.P=3 \times 10^{-8}\right)$ scores.

Spasticity, as measured by Modified Ashworth, showed modest correlations with a few task parameters: the number of objects hit with the right hand $\left(r_{s}=\right.$ -0.32, $\left.P=4.2 \times 10^{-5}\right)$, movement area with the right hand $\left(r_{s}=-0.34, P=1.6 \times 10^{-5}\right)$, and hand speed with the right hand $\left(r_{s}=-0.33, P=2.6 \times 10^{-5}\right)$.

\section{Interrater reliability}

The interrater reliability of the task parameters is shown in Table 2 for subjects (13 controls and 10 subjects with stroke) assessed in the task twice. Intraclass correlation coefficients were often high: ICC $>=0.8$, for $76 \%$ of parameters. Lower reliability values were generally associated with parameters that identified fewer subjects with stroke as impaired and thus had a relatively small range of values across the control and stroke populations. In contrast, higher reliability values tended to be associated with parameters that identified more subjects with stroke as impaired and thus tended to have a larger range of values across the inter-rater sample.

\section{Discussion}

The current study quantified impairments in stroke survivors to rapidly hit certain objects (targets) while avoiding all other objects (distractors). Up to $78 \%$ of subjects with stroke had impairments in individual global, spatial, temporal, or hand-specific task parameters. The task instructions were simple, minimizing the impact of comorbid language impairment [39]. The task was completed in $\sim 3$ min yet provided a wide range of information related to sensorimotor and cognitive function. Most parameters had high inter-rater reliability providing an objective approach to measure impairments and track recovery.

The object hit and avoid task is a variant of an object hit task in which subjects had to rapidly locate and hit all objects moving in the workspace [22]. The present task extended this approach by requiring the subject to 

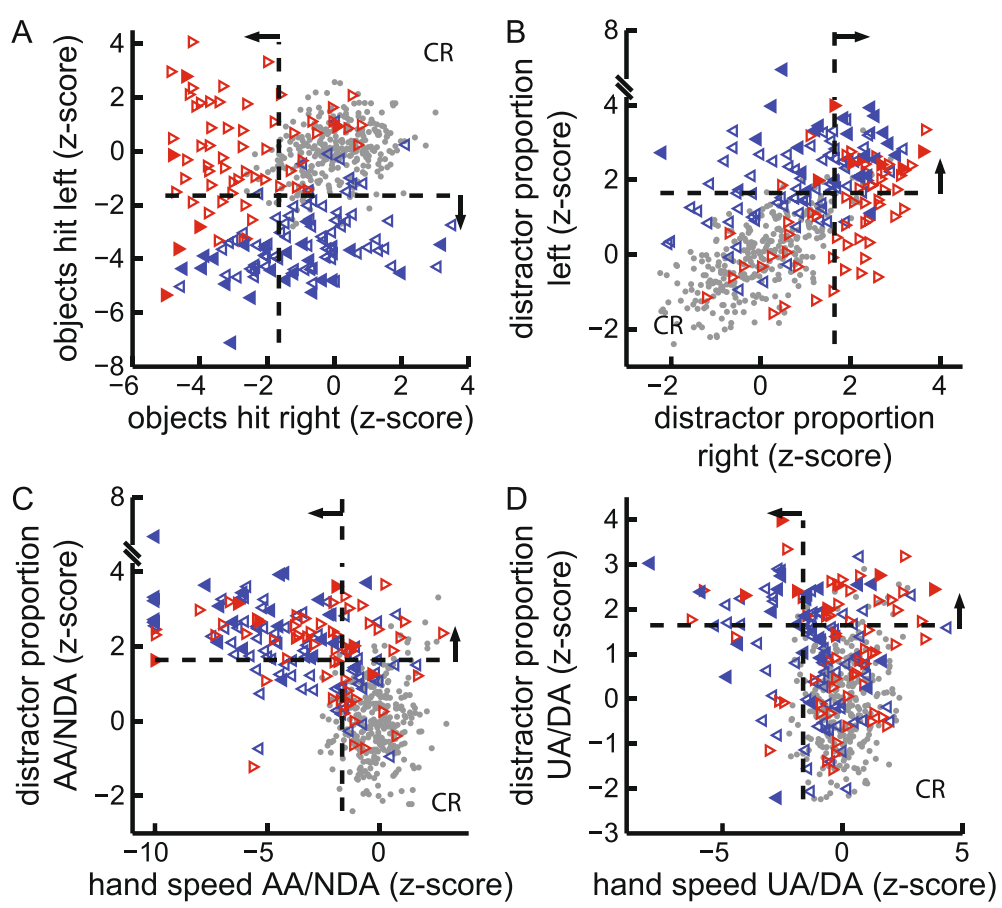

Fig. 3 Hand Specific Performance in Task Parameters. a Scatter plot of object hits (z-score) with the right versus the left hand. Symbols same as Fig. 2. $\mathbf{b}$ Scatter plot of distractor proportion with the right versus the left hand. c Scatter plot of hand speed versus distractor proportion with the affected arm (AA) of subjects with stroke and non-dominant arm (NDA) of control subjects. $\mathbf{d}$ Scatter plot of hand speed versus distractor proportion with the unaffected arm (UA) of subjects with stroke and dominant arm (DA) of control subjects

select amongst many options when moving and interacting in the environment. Total objects hit quantified each subject's ability to make rapid motor actions, regardless of whether they hit the correct objects or not. Subjects with stroke almost always hit fewer objects with their more affected side, and this arm's performance was more correlated with FIM scores than the unaffected side. Thus, the reduction and asymmetry of the ability to make rapid motor actions is quantitatively measured by the object hit and avoid task, and may have importance in the ability to complete activities of daily living.

We used a large number of parameters to quantify a broad range of sensory, motor and cognitive functions necessary to perform this task. For healthy subjects, some of these measures were highly correlated, but nevertheless captured different functions. For instance, the correlation between target hits and object hits was very strong for controls $(r=0.81)$. The reason why both parameters were measured rather than choosing only one was because it was important to differentiate between the ability to make fast and accurate movements, and the ability to make correct motor decisions om whether an object was a correct reach target or not. Thus, these metrics represent different domains of performance. Furthermore, subjects with stroke do not necessarily follow this typical pattern of performance. As shown in Fig. 2d, some subjects with stroke hit a high proportion of distractors and others do not, showing the value of each parameter to identify different impairments that do not necessarily co-occur in some individuals with stroke.

The inclusion of both target and distractor objects in the current task added an additional cognitive load to the previous object hit task. This is important as many different cognitive processes are necessary to perform daily activities, and their impairment after stroke is a significant cause of disability [40]. The present object hit and avoid task focused on a few key processes.

First, demands on the attentional system are high in a visual search task, as it requires differentiating target and distractor stimuli [41]. Rapid parallel processing of the entire visual workspace can be employed to find a target amongst many distractors with minimal effort if the target has a unique feature separate from distractors that makes it 'pop out'. In contrast, focused attention is required to serially analyze each stimulus if the target can only be differentiated from the distractors by a conjunction of features. The greater attentional demands required for a conjunction versus a feature visual search task results in greater reaction time for both controls and subjects with stroke who do not have visuospatial neglect [23]. Subjects with visuospatial neglect also show significantly increased times to detect targets in a conjunction search task (regardless of which side of the workspace was tested), when compared to the performance of controls and subjects with 
Table 3 The relationship between task performance of subjects with stroke and Functional Independence Measure (FIM), Montreal Cognitive Assessment (MoCA), and Behavioural Inattention Test (BIT) scores is shown by the corresponding Spearman correlations

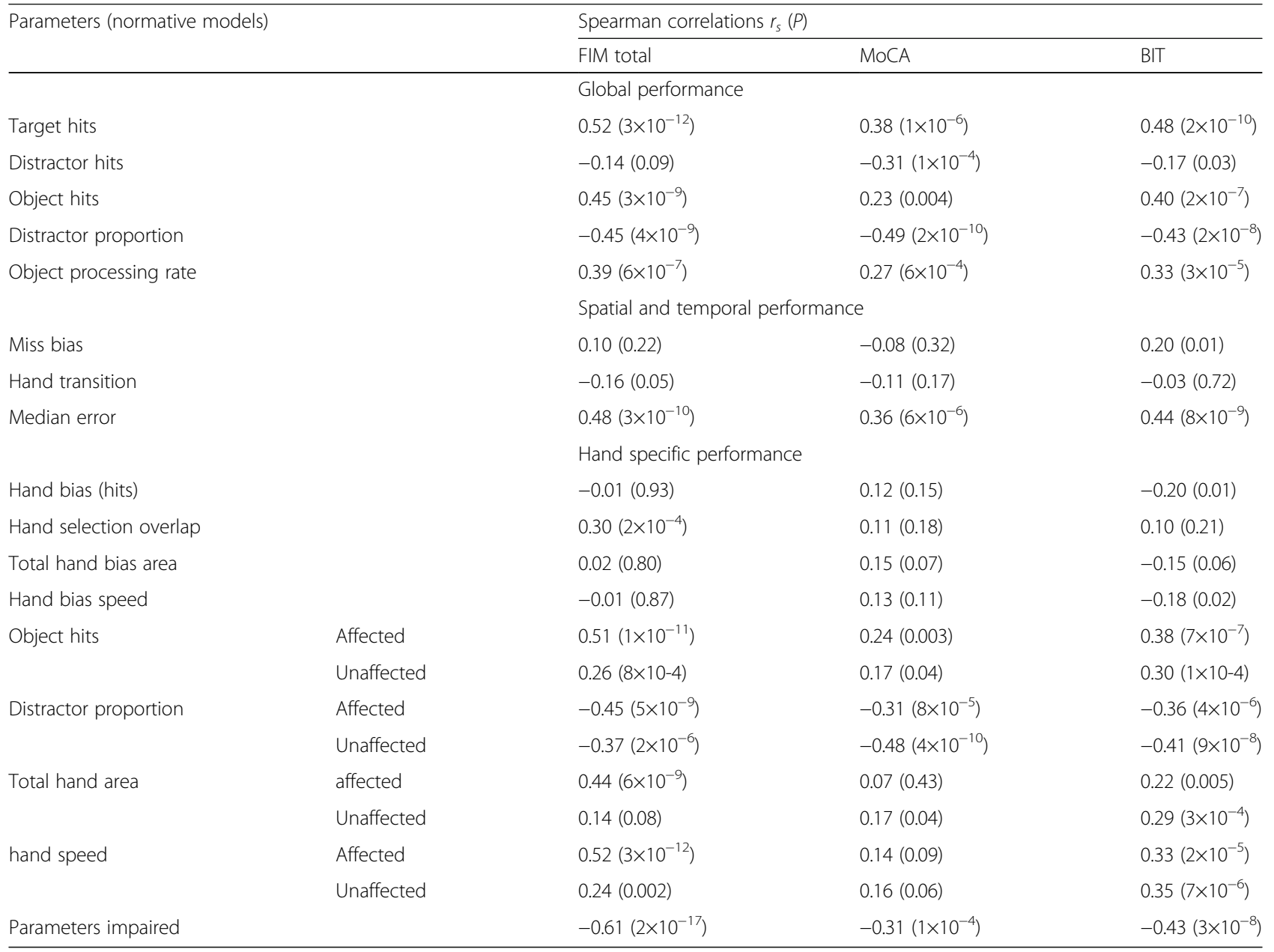

Abbreviations: $R H$ (right hand), $L H$ (left hand), LA (left-affected subject), $R A$ (right-affected subject)

stroke. The object hit and avoid task is representative of a conjunctive visual search as targets could only be differentiated from distractors by attending to the geometry (circular, three- or four-sided) and relative dimensions (tall, wide or equal) of each object (see Methods-Behavioural
Task). Correspondingly, BIT scores correlated with many individual task parameters, as well as the total number of parameters impaired. Although correlations were weak to moderate, all were in the expected direction: greater task impairment associated with greater clinical impairment.
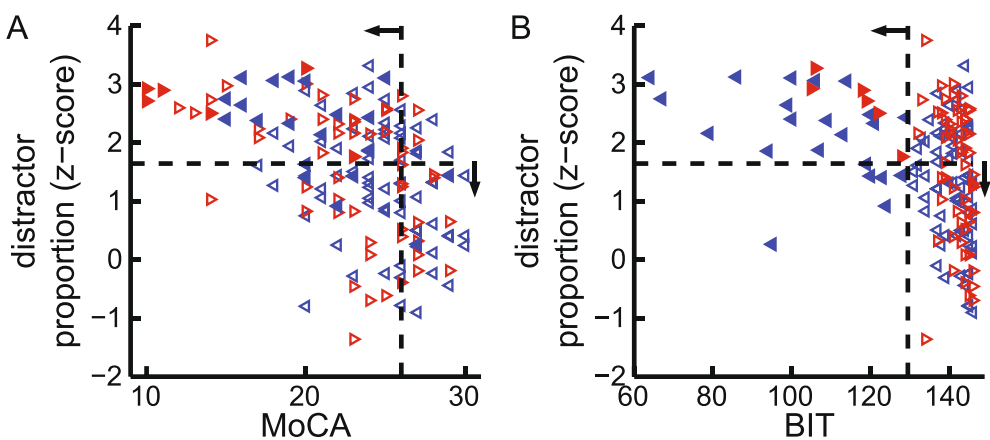

Fig. 4 Clinical correlations with task performance. a Scatter plot of Montreal Cognitive Assessment (MoCA) scores versus overall distractor proportion. Symbols same as Fig. 2. b Scatter plot of Behavioural Inattention Test (BIT) scores versus overall distractor proportion 
In the current study, participants are required to either enact a reach toward the target, or actively avoid hitting a distractor. Despite visual feedback, haptic feedback, and initial reminders on the need to hit only two types of objects and avoid the rest, over half of the subjects with stroke hit a greater proportion of distractors than $95 \%$ of controls. Subjects with stroke were twice as likely to be impaired in this parameter if they also had neglect.

The ability to inhibit a motor action is an important cognitive function of voluntary motor behaviour [7]. Motor decisional processes mediate the initiation of an automatic motor response to a new stimulus with the voluntary response required by the task [42]. This ability to inhibit stimulus-driven and enact task-driven motor responses can be measured by eye movements in the antisaccade task [43] and arm movements in an anti-pointing task [8]. In both tasks, subjects must inhibit a movement to the appearance of a visual stimulus and move to the equal and opposite location. Subjects with stroke having damage to frontal lobes have been shown to make erroneous saccades towards a stimulus in an anti-saccade task [9]. Subjects with stroke and visual neglect show greater endpoint errors and longer reaction times in an antipointing condition (on both sides of space) than controls or subjects with stroke who do not have visuospatial nelgect [24]. Distractor proportion in the current study correlated with BIT scores just as anti-pointing impairments correlated with the severity of neglect.

The assessment of rapid visuomotor skills post-stroke has potentially useful applications when rehabilitation goals are to regain high function. The object hit and avoid task may be very predictive of the ability to drive, return to work, or maintain complete independence as these skills require the ability to make many rapid motor decisions daily. We show that impairments in these skills are not always captured by currently used pen and paper cognitive screening tools such as the MoCA. Also, since this task relies on many domains of function to be successful, it may be a good indicator of overall stroke recovery.

The measurement of cognitive function after stroke, as measured by the MoCA, correlated moderately with distractor proportion, but only modestly with the number of distractor hits. As well distractor proportion also identified more subjects as impaired as compared to distractor hits. These differences reflect the fact that some control subjects hit a substantive number of distractors, but they also hit many targets. This is why we measured distractor proportion which quantified the ratio between distractors hit and total objects hit.

This task is also part of a larger research program to design a battery of robotic assessment tasks to create a quantitative diagnostic assessment of sensory, motor, and cognitive impairments post-stroke [21]. The use of a robotic assessment provides objective, continuous measures of performance that are responsive to small changes and compared to a normative model of healthy control performance. This overcomes issues of subjectivity, coarse ordinal scales, criteria-based scoring, and lack of responsiveness (including floor and ceiling effects) seen in many standard assessments of post-stroke functioning. We have also developed assessments of visually-guided reaching [44], bimanual control [30], limb position sense [26], kinesthesia [45], and limb afferent feedback for action [46]. The goal is that information from this assessment battery may be used collectively to provide more precise and responsive tools to guide individualized rehabilitation care.

Successful performance in the current task requires many sensorimotor and cognitive skills, thus failure can reflect many potential impairments in sensory, motor and cognitive functions. In order to identify unique impairments in individual participants, it is important to consider the type of parameters that show poor performance. For example, subjects who have impairments in the number objects hit, but not distractor proportion, may have underlying sensorimotor impairments, but no cognitive impairments. These subjects may be better candidates for sensorimotor rather than more cognitiverelated rehabilitation. Future work is required to identify whether these patterns of impairment can predict the best type of rehabilitation for each individual.

\section{Conclusions}

The object hit and avoid task provides a simple and fast approach to quantify the use of attention and selection to perform rapid motor actions with the arms. Most subjects with stroke were found to be impaired when performing this task, especially those with neglect. Many parameters had high inter-rater reliability and correlated with various clinical measures of impairments and ability to perform daily activities.

\section{Abbreviations \\ AA: Affected arm; BIT: Behavioural inattention test; Br: Brainstem; C: Cortical; C + SC: Cortical + subcortical; Cb: Cerebellar; Cb + Br: Cerebellar + brainstem; CMSA: Chedoke-McMaster stroke assessment; CR: Control range; DA: Dominant arm; FIM: Functional independence measure; L: Left; LA: Left-affected; LH: Left hand; M: Mixed; MoCA: Montreal cognitive assessment; Mx: Mixed; NDA: Non-dominant arm; R: Right; RA: Right-affected; RH: Right hand; SC: Subcortical; UA: Unaffected arm; Uk: (unknown)}

\section{Acknowledgements}

The authors would like to thanks S Appaqaq, H Bretzke, MJ Demers, M Metzler, K Moore, J Peterson, M Piitz, and J Yajure for their help with data collection, patient recruitment, and technical support.

\section{Funding}

This work was supported by the Ontario Research Fund - Research Excellence (ORF-RE 04-47), Canadian Institute of Health Research operating grants (MOP 106662), and the Heart and Stroke Foundation of Canada (G-13-0003029). SH Scott was supported by a GSK-CIHR chair in Neuroscience (XGG124631 \& 279791). None of these funding sources had any role in the design of the study, the collection, analysis, and interpretation of data, nor in writing the manuscript. 


\section{Availability of data and materials}

Please contact the author for data requests.

\section{Authors' contributions}

TCB assisted in data collection, and was the primary person responsible for conducting the data analysis, and writing the manuscript. CRL assisted with data analysis and drafting of the manuscript. SPD assisted with data collection and drafting of the manuscript. SDB assisted with data collection and drafting of the manuscript. KEN assisted with data analysis and drafting of the manuscript. SHS participated in the design of the study, data analysis, and drafting of the manuscript. All authors read and approved the final manuscript.

\section{Competing interests}

SHS is co-founder and chief scientific officer of BKIN Technologies that commercializes the KINARM robot.

\section{Consent for publication}

Not applicable.

\section{Ethics approval and consent to participate}

This study was approved by the Queen's University Health Sciences and Affiliated Teaching Hospitals Research Ethics Board (\#ANAT-024-05) and the University of Calgary's Conjoint Health Research Ethics Board (\#22123) and subjects provided informed consent.

\section{Author details}

'Centre for Neuroscience Studies, Queen's University, Kingston, ON, Canada. ${ }^{2}$ Department of Physical Medicine and Rehabilitation, Queen's University, Kingston, ON, Canada. ${ }^{3}$ School of Rehabilitation Therapy, Queen's University, Kingston, ON, Canada. ${ }^{4}$ Department of Biomedical and Molecular Sciences, Queen's University, Kingston, ON, Canada. ${ }^{5}$ Department of Clinical Neurosciences, Hotchkiss Brain Institute, University of Calgary, Calgary, AB, Canada.

\section{Received: 3 March 2016 Accepted: 4 October 2016}

\section{Published online: 10 October 2016}

\section{References}

1. Cisek P. Cortical mechanisms of action selection: the affordance competition hypothesis. Philos Trans R Soc Lond B Biol Sci. 2007;362:1585-99.

2. Cisek P, Pastor-Bernier A. On the challenges and mechanisms of embodied decisions. Philos Trans R Soc Lond B Biol Sci. 2014;369.

3. Scott SH. A functional taxonomy of bottom-up sensory feedback processing for motor actions. Trends Neurosci. 2016:39:512-26.

4. Treue S. Neural correlates of attention in primate visual cortex. Trends Neurosci. 2001:24:295-300.

5. Tanaka K, Saito H, Fukada Y, Moriya M. Coding visual images of objects in the inferotemporal cortex of the macaque monkey. J Neurophysiol. 1991;66:170-89.

6. Eskandar EN, Richmond BJ, Optican LM. Role of inferior temporal neurons in visual memory. I. Temporal encoding of information about visual images, recalled images, and behavioral context. J Neurophysiol. 1992;68:1277-95.

7. Munoz DP, Everling S. Look away: the anti-saccade task and the voluntary control of eye movement. Nat Rev Neurosci. 2004;5:218-28.

8. Day BL, Lyon IN. Voluntary modification of automatic arm movements evoked by motion of a visual target. Exp Brain Res. 2000;130:159-68.

9. Guitton D, Buchtel HA, Douglas RM. Frontal lobe lesions in man cause difficulties in suppressing reflexive glances and in generating goal-directed saccades. Exp Brain Res. 1985;58:455-72.

10. Pierrot-Deseilligny C, Muri RM, Ploner CJ, Gaymard B, Demeret S, RivaudPechoux S. Decisional role of the dorsolateral prefrontal cortex in ocular motor behaviour. Brain. 2003;126:1460-73.

11. Hawkins KM, Sayegh P, Yan X, Crawford JD, Sergio LE. Neural activity in superior parietal cortex during rule-based visual-motor transformations. J Cogn Neurosci. 2013;25:436-54.

12. Tippett WJ, Alexander LD, Rizkalla MN, Sergio LE, Black SE. True functional ability of chronic stroke patients. J Neuroeng Rehabil. 2013;10:1.

13. Salek $Y$, Anderson ND, Sergio L. Mild cognitive impairment is associated with impaired visual-motor planning when visual stimuli and actions are incongruent. Eur Neurol. 2011;66:283-93.

14. Tippett WJ, Sergio LE. Visuomotor integration is impaired in early stage Alzheimer's disease. Brain Res. 2006;1102:92-102.
15. Brown JA, Dalecki M, Hughes C, Macpherson AK, Sergio LE. Cognitive-motor integration deficits in young adult athletes following concussion. BMC Sports Sci Med Rehabil. 2015;7:1.

16. Teasell R, Hussein N. Clinical consequences of stroke. In: Evidence based review of stroke rehabilitation (wwwebrsrcom). 2013.

17. Salter K, Campbell N, Richardson M, Mehta S, Jutai J, Zettler L, Moses M, McClure A, Mays R, Foley N, Teasell R. Outcome measures in stroke rehabilitation. In: Evidence Based Review of Stroke Rehabilitation (wwwebrsrcom). 2013.

18. Cumming TB, Brodtmann A, Darby D, Bernhardt J. Cutting a long story short: reaction times in acute stroke are associated with longer term cognitive outcomes. J Neurol Sci. 2012;322:102-6.

19. Ballard C, Stephens S, Kenny R, Kalaria R, Tovee M, O'Brien J. Profile of neuropsychological deficits in older stroke survivors without dementia. Dement Geriatr Cogn Disord. 2003;16:52-6.

20. Sanchez-Cubillo I, Perianez J, Adrover-Roig D, Rodriguez-Sanchez J, RiosLago M, Tirapu J, Barcelo F. Construct validity of the trail making test: role of task-switching, working memory, inhibition/interference control, and visuomotor abilities. J Int Neuropsychol Soc. 2009:15:438.

21. Scott SH, Dukelow SP. Potential of robots as next-generation technology for clinical assessment of neurological disorders and upper-limb therapy. J Rehabil Res Dev. 2011:48:335-53.

22. Tyryshkin K, Coderre AM, Glasgow Jl, Herter TM, Bagg SD, Dukelow SP, Scott $\mathrm{SH}$. A robotic object hitting task to quantify sensorimotor impairments in participants with stroke. J Neuroeng Rehabil. 2014;11:47.

23. Erez $A B$, Katz N, Ring H, Soroker N. Assessment of spatial neglect using computerised feature and conjunction visual search tasks. Neuropsycho Rehabil. 2009:19:677-95.

24. Rossit S, Malhotra P, Muir K, Reeves I, Duncan G, Harvey M. The role of right temporal lobe structures in off-line action: evidence from lesion-behavior mapping in stroke patients. Cereb Cortex. 2011;21:2751-61.

25. Scott SH. Apparatus for measuring and perturbing shoulder and elbow joint positions and torques during reaching. J Neurosci Methods. 1999;89:119-27.

26. Dukelow SP, Herter TM, Moore KD, Demers MJ, Glasgow JI, Bagg SD, Norman KE, Scott SH. Quantitative assessment of limb position sense following stroke. Neurorehabil Neural Repair. 2010:24:178-87.

27. von Bertalanffy L. On the von Bertalanffy growth curve. Growth. 1966;30:123-4.

28. Allen KR. A method of fitting growth curves of the von bertalanffy type to observed data. J Fish Res Board Can. 1966;23:163-79.

29. Cormen TH, Leiserson CE, Rivest RL. Introduction to algorithms. Cambridge: MIT press; 1990

30. Lowrey C, Jackson C, Bagg S, Dukelow S, Scott S. A novel robotic task for assessing impairments in bimanual coordination post-stroke. Int J Phys Med Rehabil S. 2014;3:2

31. Box GE, Cox DR. An analysis of transformations. J R Stat Soc B Methodol. $1964 ; 211-252$

32. Gowland C, Stratford P, Ward M, Moreland J, Torresin W, Van Hullenaar S, Sanford J, Barreca S, Vanspall B, Plews N. Measuring physical impairment and disability with the chedoke-McMaster stroke assessment. Stroke. 1993;24:58-63.

33. Brunnstrom S. Motor testing procedures in hemiplegia: based on sequential recovery stages. Phys Ther. 1966:46:357.

34. Bohannon RW, Smith MB. Interrater reliability of a modified Ashworth scale of muscle spasticity. Phys Ther. 1987;67:206-7.

35. Keith RA, Granger CV, Hamilton BB, Sherwin FS. The functional independence measure: a new tool for rehabilitation. Adv Clin Rehabil. 1987:1:6-18.

36. Wilson B, Cockburn J, Halligan P. Development of a behavioral test of visuospatial neglect. Arch Phys Med Rehabil. 1987;68:98-102.

37. Nasreddine ZS, Phillips NA, Bedirian V, Charbonneau S, Whitehead V, Collin I, Cummings $\mathrm{J}$, Chertkow $\mathrm{H}$. The Montreal Cognitive Assessment, MoCA: a brief screening tool for mild cognitive impairment. J Am Geriatr Soc. 2005:53:695-9.

38. Oldfield RC. The assessment and analysis of handedness: the Edinburgh inventory. Neuropsychologia. 1971:9:97-113.

39. Pasi M, Salvadori E, Poggesi A, Inzitari D, Pantoni L. Factors predicting the Montreal cognitive assessment (MoCA) applicability and performances in a stroke unit. J Neurol. 2013;260:1518-26.

40. Cicerone KD, Dahlberg C, Kalmar K, Langenbahn DM, Malec JF, Bergquist TF, Felicetti T, Giacino JT, Harley JP, Harrington DE. Evidence-based cognitive rehabilitation: recommendations for clinical practice. Arch Phys Med Rehabil. 2000;81:1596-615.

41. Treisman AM, Gelade G. A feature-integration theory of attention. Cogn Psychol. 1980;12:97-136. 
42. Theeuwes J, Kramer AF, Hahn S, Irwin DE. Our eyes do not always go where we want them to go: capture of the eyes by new objects. Psychol Sci. 1998;9:379-85.

43. Hallett PE. Primary and secondary saccades to goals defined by instructions. Vision Res. 1978;18:1279-96.

44. Coderre AM, Zeid AA, Dukelow SP, Demmer MJ, Moore KD, Demers MJ, Bretzke H, Herter TM, Glasgow II, Norman KE, et al. Assessment of upper-limb sensorimotor function of subacute stroke patients using visually guided reaching. Neurorehabil Neural Repair. 2010;24:528-41.

45. Semrau JA, Herter TM, Scott SH, Dukelow SP. Robotic identification of kinesthetic deficits after stroke. Stroke. 2013;44:3414-21.

46. Bourke TC, Coderre AM, Bagg SD, Dukelow SP, Norman KE, Scott SH. Impaired corrective responses to postural perturbations of the arm in individuals with subacute stroke. J Neuroeng Rehabil. 2015;12:7.

Submit your next manuscript to BioMed Central and we will help you at every step:

- We accept pre-submission inquiries

- Our selector tool helps you to find the most relevant journal

- We provide round the clock customer support

- Convenient online submission

- Thorough peer review

- Inclusion in PubMed and all major indexing services

- Maximum visibility for your research

Submit your manuscript at www.biomedcentral.com/submit
Biomed Central 